
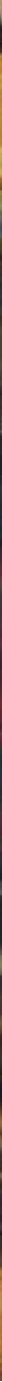

Wolfgang Krodel d. Ä., Gesetz und Gnade, 1542, Öl auf Holz, Klosterkirche und Sakralmuseum St. Annen, Kamenz, Dauerleihgabe der Evangelisch-Lutherischen Kirchgemeinde Kamenz Foto: Dietmar Träupmann, Augustusburg

\section{"Gesetz und Gnade" von Wolfgang Krodel d. Ä.}

\author{
Ein Meisterwerk der Reformation
}

im Kamenzer Sakralmuseum St. Annen

\title{
Sören Fischer
}

Im Zuge des Reformationsjubiläums 2017 konnte das Kamenzer Sakralmuseum St. Annen (Städtische Sammlungen Kamenz) erstmals ein Gemäldepaar aus seinem Bestand in den Mittelpunkt rücken, in Zusammenarbeit mit dem Cranach Digital Archive (Düsseldorf/Dresden) gemäldetechnisch untersuchen lassen und der Öffentlichkeit im Rahmen einer Sonderausstellung sowie eines ausführlichen Katalogs umfassend vorstellen, das aufgrund seines ausgeklügelten Bildprogramms $\mathrm{zu}$ den herausragenden Kunstwerken der Reformation in Sachsen zählt.

Es handelt sich dabei um das Bild „Gesetz und Gnade“, das der vermutlich im sächsischen Schneeberg geborene Maler Wolfgang Krodel der Ältere (1500-
1563) im Jahr 1542 signiert und datiert hat. Krodel, dessen Werke heute u.a. im Landesmuseum Darmstadt sowie im Kulturhistorischen Museum in Wien aufbewahrt werden, hatte zu dieser Zeit seine Ausbildung beim Wittenberger Meister Lucas Cranach dem Älteren (um 1472-1553) bereits erfolgreich abgeschlossen und sich selbstständig in Schneeberg niedergelassen. Mit „Gesetz und Gnade“ nahm er direkt auf Cranach Bezug, interpretierte dessen Stil behutsam und schuf eine feinmalerisch, exquisit ausgeführte Version des geradezu revolutionären lutherischen Grundgedankens von der Erlösung des sündigen Menschen allein aus Glauben.

Keine andere Bilderfindung der Reformation steht so prägnant für die neue lutherische Lehre 
wie „Gesetz und Gnade“, keine wurde in Form von Flugblättern, Buchpublikationen und Malerei häufiger verbreitet. „Gesetz und Gnade“ ist ein Thema, das zwischen religiöser Verheißung und politischer Polemik oszilliert und zu den hervorstechenden Bilderfindungen der Renaissance zählt. Dass das Kamenzer Sakralmuseum die einzige derart eng an Cranach orientierte Fassung von „Gesetz und Gnade“ innerhalb der sächsischen Museumslandschaft bewahrt, ist für das kommunale, 2011 in einer immer noch geweihten evangelischen Kirche eröffnete Museum Privileg und Verpflichtung zugleich.

Eindrücklich und didaktisch nachvollziehbar übertrug „Gesetz und Gnade“ Luthers Lehre der Erlösung des Menschen vom komplexen theologischen Text in das Medium des Bildes. Der Reformator war bereits in den Anfangsjahren seiner Wittenberger Professur auf der Suche nach dem gnädigen Gott. Damit verfolgte er zugleich ein Ziel, das dem damaligen theologischen Weltbild der römischen Kirche diametral entgegenstand. In diesem nämlich waren Tod, Teufel und Angst sehr viel stärker vertreten als die freudige Erwartung auf das Paradies. So wurde die Jenseitsvorstellung der Menschen im ausgehenden Mittelalter nicht von der Gewissheit auf das erfreuliche ewige Leben bestimmt, sondern von der Angst einer dem Tod folgenden mehr oder weniger langen Zeit der Qualen im Fegefeuer und in der Hölle. Das Feuer sollte den armen Seelen im nachtodlichen Reinigungsort die Sündenmakel abbrennen. Diese schreckliche Zukunftsvision fand dann auch Eingang in die Bildproduktion und wurde den Gläubigen beispielsweise in den kirchlichen Portalreliefs mit Darstellungen des Jüngsten Gerichts warnend und abschreckend vor Augen geführt.

In diese geistige Welt der Jenseitsungewissheit wurde am 10. November 1483 Martin Luther hineingeboren. Wie kaum ein anderer Theologe seiner Zeit trieb ihn die Frage nach dem Schicksal des Menschen und seiner Seele nach dem Tode um. Dies führte ihn zu folgender Einsicht: Alle Menschen sind vor Gott Sünder, da sie, auch wenn sie es wollten und noch so sehr versuchten, die biblischen Gesetze nicht vollständig befolgen können. Entsprechend dem mittelalterlichen Weltbild war also allen Menschen das Strafgericht Gottes gewiss. Für Luther war dies, wie er in seiner 1545 verfassten Vorrede zum ersten Band der Wittenberger Ausgabe seiner lateinischen Schriften formulierte, eine existentielle Erkenntnis, die ihm von Gott entfremdete: „Ich konnte den gerechten, die Sünder strafenden Gott nicht lieben, im Gegenteil, ich hasste ihn sogar. Wenn ich auch als Mönch untadelig lebte, fühlte ich mich vor Gott doch als Sünder, und mein Gewissen quälte mich sehr. Ich wagte nicht zu hoffen, dass ich Gott durch meine Bußfertigkeit versöhnen könne.“

Den Schlüssel für die Erlösung des Menschen aus dem Zustand der Sünde ohne die Voraussetzung von Bußetaten lieferte Luther dann der
Brief des Apostels Paulus an die Römer. Mit diesem hatte er sich spätestens bereits ab 1515 beschäftigt. Sein besonderes Augenmerk richtete Paulus auf die Frage, wie der einzelne Mensch durch einen barmherzigen Gott Gnade finden könne. Unter Römer 3, 22-26 liest man: „Ich rede aber von der Gerechtigkeit vor Gott, die da kommt durch den Glauben an Jesus Christus zu allen, die glauben. Denn es ist hier kein Unterschied: Sie sind allesamt Sünder und ermangeln des Ruhmes, den sie vor Gott haben sollen, und werden ohne Verdienst gerecht aus seiner Gnade durch die Erlösung, die durch Christus Jesus geschehen ist."

Für Luther bot die Exegese dieser Bibelstelle den Schlüssel für die Annäherung an den gnädigen Gott, sie bildete die eigentliche Grundlage für die sogenannte reformatorische Wende, d. h. die Formulierung von der Erlösung des Menschen „allein aus Gnade“ („sola gratia“) und „allein aus Glauben“ („sola fide“), ab etwa 1515 , spätestens aber ab 1518. Ein Jahr nach dem Verfassen der 95 Thesen veröffentlichte Luther bereits die Schrift „De remissione peccatorum“ (,Von der Vergebung der Sünden“). Dort berief er sich wörtlich auf Paulus: „Wenn er [der Mensch] an den übrigen [Sünden] verzweifelt, muss er sich selbst mit Zuversicht in den Abgrund der Barmherzigkeit Gottes werfen, der die Barmherzigkeit treulich zugesagt hat. Der Gerechte wird nicht aus Werken des Gesetzes, auch nicht aus dem Gesetz, sondern aus Glauben leben.“ Damit war die Rechtfertigungslehre als Grundpfeiler der neuen lutherischen Lehre formuliert. Allein der Christusglaube, so Luther, mache den Menschen vor Gott gerecht, allein das Vertrauen in Jesus löse von der Angst vor dem brennenden Fegefeuer - Heiligenverehrung, Ablassbriefe etc. waren damit unnötig geworden; ein radikaler Angriff auf die scholastische Sündenlehre der römisch-katholischen Kirche. Für die Reformation entwickelte sich die Rechtfertigungslehre zum theologischen Argumentationskern. 1537 schrieb Luther: „Der Artikel von der Rechtfertigung ist Meister und Fürst, Herr, Leiter und Richter über alle Arten von Lehren, der alle kirchlichen Lehren bewahrt und lenkt und unser Gewissen vor Gott aufrichtet.“ Dass sich die Rechtfertigungslehre rasch im europäischen Kulturraum verbreiten konnte, ist dabei maßgeblich dem Medium des Bildes zu verdanken. Schon Ende der 1520er Jahre nämlich hatte Lucas Cranach d. Ä., der als berühmter Hofmaler zugleich zum engen Wittenberger Freundeskreis Luthers zählte, erste bildliche Interpretationen der Rechtfertigungslehre entworfen. 1529 entstand beispielsweise das kleinformatige Gemälde „Gesetz und Gnade“, das heute in Schloss Friedenstein in Gotha aufbewahrt wird. Ihm folgten neben weiteren Gemäldeversionen (heute $u$. a. in Prag und Weimar) der 1539 fertiggestellte Altar der St.-Wolfgangs-Kirche in Schneeberg sowie zahlreiche Buch- bzw. Flugblattillustrationen. In Wittenberg, spätestens aber in Schneeberg wird 
Lucas Cranach d. Ä., Gesetz und Gnade, Öl auf Holz, 1529, Stiftung Schloss Friedenstein, Gotha, Inv.-Nr. SG 676 ๑ Stiftung Schloss Friedenstein, Gotha auch Krodel mit dem Thema in Berührung gekommen sein. Vielleicht - die dünne Überlieferungslage lässt bisher nur Spekulationen zu - war das Gemäldepaar, das sich heute im Eigentum der Evangelisch-Lutherischen Kirchgemeinde $\mathrm{Ka}-$ menz befindet, ein Auftragswerk des lutherisch orientierten Kamenzer Stadtrates.

In Übereinstimmung mit den Cranach'schen Vorbildern teilte auch Krodel das Bild entsprechend einem typologischen Aufbau in zwei Bereiche, welche die Zeit des Gesetzes (links: Altes Testament) und die der Gnade (rechts: Neues Testament) allegorisch darstellten. Ein Baum, der auf der linken Seite kahl und tot ist, auf der rechten Seite aber blüht, bildet in diesem reformatorischen Bekenntnisbildes das zentrale Verbindungslied. Bibelzitate in deutscher Sprache erläutern dabei im unteren Register die jeweils dargestellten Szenen und untermauern zugleich den theologischen Gehalt des Bildes.

Im Zentrum der linken Tafel steht ein fast unbekleideter Mann, der als Adam die sündige Menschheit repräsentiert; ein „Jedermann“ und damit für den Betrachter eine perfekte Identifikationsfigur. In panischer Fluchtbewegung hat er die Augen weit aufgerissen und die Arme hilfesuchend gen Himmel gerissen. Verzweiflung durchströmt ihn, weil er erkennt, dass er als Mensch nicht nur Nachfolger der Erbsünde ist (man beachte Adam und Eva auf dem Hügel im Hintergrund), sondern aufgrund seiner Schwächen auch die Gebote Gottes nicht vollständig befolgen kann, und also verdammt ist. Dies wird ihm mit einem Blick auf Mose und die Gesetzestafeln einmal mehr deutlich. Ohne Hoffnung auf Erlösung und verfolgt von Tod und Teufel - diese traktieren ihn bereits mit Spieß und Krallen steht er am Abgrund zur Hölle, deren Flammen ihm quälend entgegenschlagen.

Das Gemäldepaar löst diesen unerträglichen $\mathrm{Zu}$ stand im Sinne Luthers mit der rechten Bildtafel, der Seite der Gnade, nun erlösend und didaktisch gelungen auf. Diese setzt das Lutherwort von der Rechtfertigung kongenial ins Bild: „Die wahre Reue kommt nicht aus uns, sondern aus der Gnade Gottes; deshalb müssen wir an uns verzweifeln und zu seiner Barmherzigkeit $\mathrm{Zu}$ flucht nehmen." Entsprechend diesem Postulat aus „Sermo de Poenitentia“ (1518) folgt der nackte, nun beruhigte Sünder dem Wort und Fingerzeig von Johannes dem Täufer und nimmt den Gekreuzigten als seinen Erlöser an. Dem Menschen ist durch sein Gottvertrauen jede Angst vor dem Tod genommen. Der Blutstrahl, der von Christus auf den nackten Adam zielt, macht abschließend sinnbildlich, dass sich das

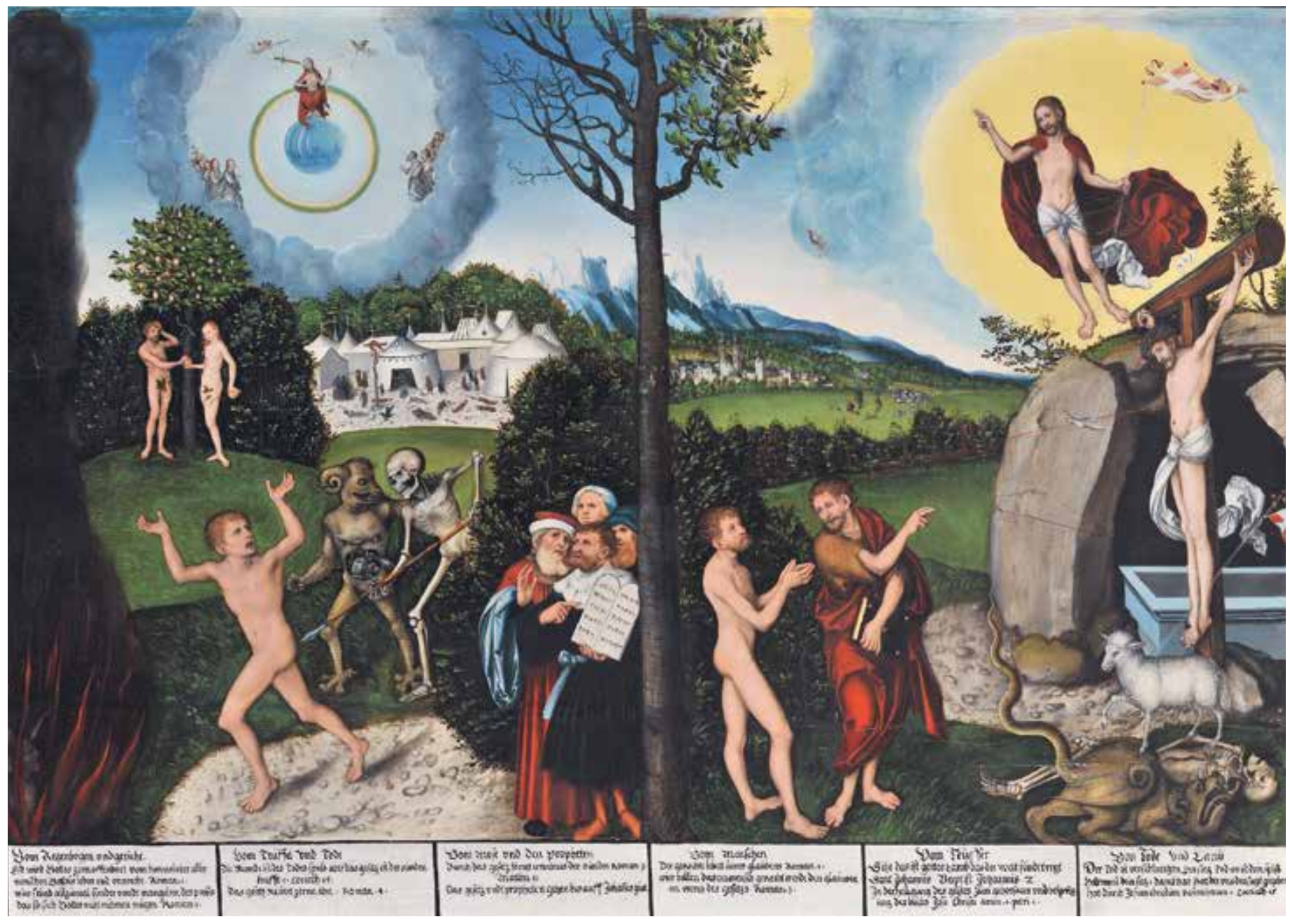


Kreuzesopfer (prophezeit bereits in der Marienvision auf dem Hintergrundberg) und die ihm folgende Auferstehung (diese wird durch den triumphierenden und Tod und Teufel besiegenden Christus auf der rechten Seite verkörpert) „allein aus Glauben“ auf den Menschen überträgt - ein Versprechen, das dem Individuum im Diesseits Seelenruhe bescherte und die Angst vor dem Tod zumindest ein Stück weit mildern konnte. Nun erklärt sich auch die alttestamentliche Zeltstadt im Hintergrund der linken Tafel: Dort wird nach 4. Mose 21, 4-9 geschildert, wie Gott zuerst die aus Ägypten ausgezogenen und unzufriedenen Israeliten durch Schlangenbisse strafte, um seinem Volk dann mit einer Schlange aus Erz ein Zeichen der Erlösung zu schicken. Ebenso wie der Adam auf der Seite der "Gnade“ wurden die Israeliten also allein durch den Anblick der Schlange und ihren Glauben an die Erlösungskraft Gottes errettet.

Krodel orientierte sich bei seiner Interpretation eng an Cranach, wobei neben dem Altar der St.Wolfgangs-Kirche wohl auch Holzschnitte wie die der erstmals 1541 gedruckten sogenannten Lufft-Bibel (Medianbibel) als Vorlagen dienten. Es spricht jedoch zugleich für die künstlerische Kreativität Krodels, dass er sich in einem wichtigen Bilddetail vom Malerfürsten abhob. Der Schneeberger Künstler nämlich erweiterte sein Werk um eine plakative Polemik gegen das Oberhaupt der römischen Kirche. Die Rede ist von der Teufelsgestalt, die mit scharfen Pranken, Hahnenschnabel, Eselsohren und Greifenfüßen eine direkte Ausgeburt der Hölle zu sein

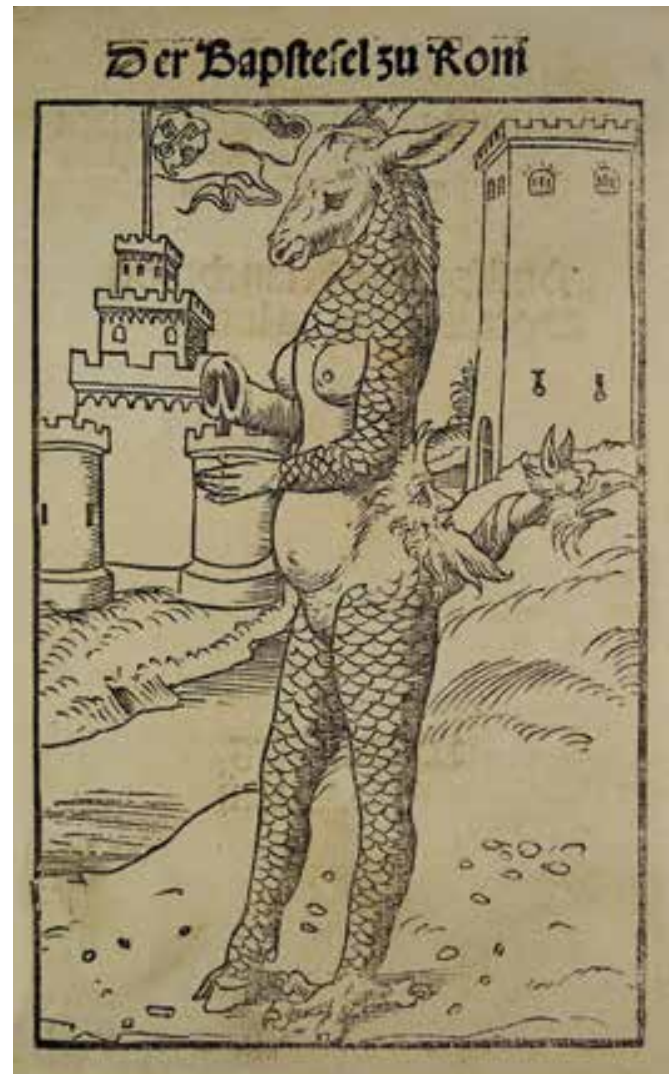

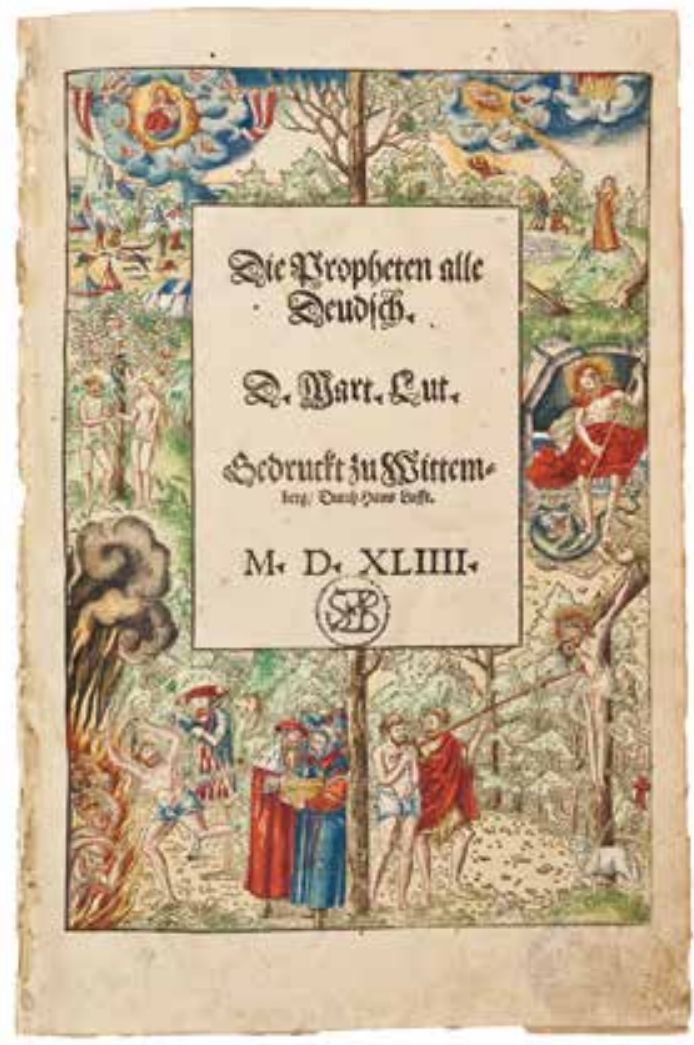

scheint. Bereits frühere Fassungen von „Gesetz und Gnade“ hatten der Teufelsgestalt eine besondere Aufmerksamkeit gewidmet. Es war aber erstmals Krodel, der die damalige Auseinandersetzung mit dem Papst derart unzweideutig in eine Gemäldefassung von „Gesetz und Gnade“ übertrug.

Karikaturen des kirchlichen Oberhauptes waren in der Reformation bekanntlich ein integraler Bestandteil des ideologischen Kampfes. Exemplarisch verdeutlicht dies die Darstellung des sogenannten „Papstesels“, die in der CranachWerkstatt entstand und das Oberhaupt als abnormales Mischwesen verunglimpfte. Krodel setzte hier an und krönte seine Teufelsgestalt mit einer der Tiara ähnlichen Kopfbedeckung. Dass der Teufel darüber hinaus noch ein Pallium trägt, kennzeichnet ihn abschließend als Vertreter der römischen Kirche. Cranach d. Ä. hatte es noch vermieden, seinen Gemäldefassungen diese Schärfe zu geben. Krodel hingegen verband das theologische Lehrbild mit einer deutlichen Kennzeichnung des Pontifex als Gegner und Antichrist; möglicherweise eine direkte Reaktion auf die Schmalkaldischen Artikel von $1536 / 37$ oder aber individueller Wunsch der Auftraggeber.

Nicht nur aufgrund dieser singulären polemischen Note muss Krodels Gemäldepaar „Gesetz und Gnade“ als herausragendes künstlerisches Zeugnis der Reformation in Sachsen bezeichnet werden. In der Dauerausstellung des Sakralmuseums St. Annen präsentiert, ist es zugleich ein Meisterwerk und Publikumsmagnet der Lessingstadt wie des Museums.
Werkstatt Lucas Cranachs d. Ä., Gesetz und Gnade, 1544; Holzschnitt, koloriert, Titelblatt, aus: Martin Luther: Die Propheten alle Deudsch [Biblia. Das ist. Die gantze Heilige Schrift], Wittenberg (Lufft), 1544 ๑ Christian-Weise-Bibliothek Zittau

Werkstatt Lucas Cranachs d. Ä., Der Papstesel, 1523, Holzschnitt aus: Philipp Melanchthon/Martin Luther: Deuttung der zwo grewlichen Figuren Bapstesels zu Rom vnd Munchkalbs zu freyberg jn Meyssen funden, Wittenberg 1523

๑ Oberlausitzische Bibliothek der Wissenschaften, Görlitz

\section{Autor}

Dr. Sören Fischer

Kurator des Sakralmuseums

St. Annen

Städtische Sammlungen

Kamenz

Schulplatz 5, 01917 Kamenz soeren.fischer@stadt.kamenz.de 